\title{
Multi-Body Finite Element Analysis of an Inclined Scrap Shear with 1100 Tons of Capacity
}

\author{
Hira Karagulle $^{1}$, Cagatay Y. Yildırım², Murat Akdag ${ }^{1}$, \\ Ramazan San ${ }^{2}$, Hayri Yildiz ${ }^{1}$, Levent Malgaca ${ }^{1}$, Sahin Yavuz ${ }^{1}$ \\ ${ }^{1}$ Dept. of Mechanical Eng., Dokuz Eylul University, İzmir, Turkey \\ ${ }^{2}$ Research and Development Center, A YMAS Makine Ltd., İmir, Turkey
}

\begin{abstract}
Engineering analysis of a commercial inclined scrap shear with a cutting force of $\mathbf{1 1 0 0}$ tons is given in this study. An analysis model is created in Solidworks (SW). The assembly of the system contains members, which are the frame, two flippers, clamp, and guillotine. There are a revolute joint between the flippers and the frame, a slider joint between the clamp and the frame, and a slider joint between the guillotine and the frame. The free-body diagrams of the members are explored. Multi-body finite element (FE) analysis of the whole assembly is used instead of single-body FE analysis of members. A pin connection is defined for the revolute joint and, no penetration contacts are defined for the slider joints in the multi-body analysis. The fixed boundary condition is defined on the surface of the frame contacting the ground. The moving members are actuated by the fluid power with $\mathbf{3 0 0}$ bars of maximum pressure. The flippers and the clamp press the scrap metal and the guillotine cuts it. The curvature-based mesh is used in FE analysis. The von Mises stresses and deformations are calculated. Smaller mesh sizes are used by using mesh control in the critical regions where high stresses occur to check the stability of the results. Average stress values are considered in the regions where the maximum stress occurs at single finite elements.
\end{abstract}

Keyword- Finite element, multi-body, recycling machines, Solidworks

\section{INTRODUCTION}

Hydraulic scrap shears are used in the metal recycling industries to press and cut long and large volumes of scrap into usable sizes. Scrap shears are classified according to their production capacity and shear force, which are important design criteria. Inclined scrap shears benefit from the force of gravity to feed the material more easily. The material can be easily fed by gravity using less energy. Inclined scrap shear from 670 ton to 1375 ton is available in the markets (Aymas, Metso, 2019). The operating principle of scrap shears can be considered in three steps. In the first step, the side flippers compress the material laterally. In the second step, the upper clamp compresses the material vertically. In the last step, the guillotine cuts the material at the desired cutting length. The cutting material is held between the guillotine and the retention flap which adjusts the cutting length of the material.

Large forces occur during compression and cutting operations. These forces are transmitted to the frame. The frame must be designed to withstand these forces. The design is done by evaluating the stress and deformation results in critical regions.

The FE method provides a powerful solution for the analysis of such complex structures or machines. Machines usually consist of assembly of members. Assembled systems can be analyzed with CAE tools such as SW, ANSYS and Proengineer. FE analysis of assembled systems is an engineering problem and requires great challenges. Assumptions such as joints, contacts and boundary conditions defined on such assembled systems affect the results. The modeling approach and meshing should be determined so as to shorten the analysis time and therefore the design process in general.

The analysis of each member in the assembly can be performed separately. For this purpose, free-body diagrams must be drawn and the forces transmitted to each member must be determined using the laws of mechanics. However, here the values of the forces and the points on which the forces act must not be nonlinear and unclear.

The literature on the design of scrap shears is very limited. Studies on shearing and guillotine systems are summarized here. (Ramamurti et al., 1997) presented the dynamic analysis of a straight guillotine-shearing machine with the FE method. They examined the vibration and strength characteristics of the machine and blade carrier due to the change in plate thickness. (Wang et al., 2010) analyzed a hydraulic shear's frame and presented the results of its deformation, equivalent stress and strain.

There are studies on modeling and analysis of guillotining process. (Bohdal and Kukielka, 2014) studied the modelling, analysis, and testing procedure for guillotining process of a sheet metal. They presented simulations using ANSYS/LSDYNA and experimental verifications. (Wisselink and Hu'etink, 2004) developed a FE model of guillotining to calculate the steady state of such a process using the Arbitrary Lagrangian Eulerian formulation. (Liu et al., 2009) analyzed shear force according to uniform load ANSYS APDL and obtained the deformation, stress and strain of the tool carrier on moving load.

In this work, multi-body FE analysis of a commercial inclined scrap shear with a cutting force of 1100 tons instead of single-body FE analysis of members is studied in SW. Maximum working pressure is considered as 300 bars for the hydraulic actuation. The forces acting on the assembly are calculated according to the maximum hydraulic pressure. Critical regions are determined on the system by evaluating the VonMises stresses and deformations. The effect of mesh size and singularity are examined in the region where high stress occurs. 


\section{SYSTEM UNDER STUDY}

The system under study is shown in Fig. 1. The SW analysis model where the parts carrying high loads are considered is shown in Fig. 2. The analysis model does not include the details of the production model. It is used to evaluate the thicknesses of the plates. The hydraulic cylinders are evaluated by using conventional engineering methods, which are not presented in this paper.

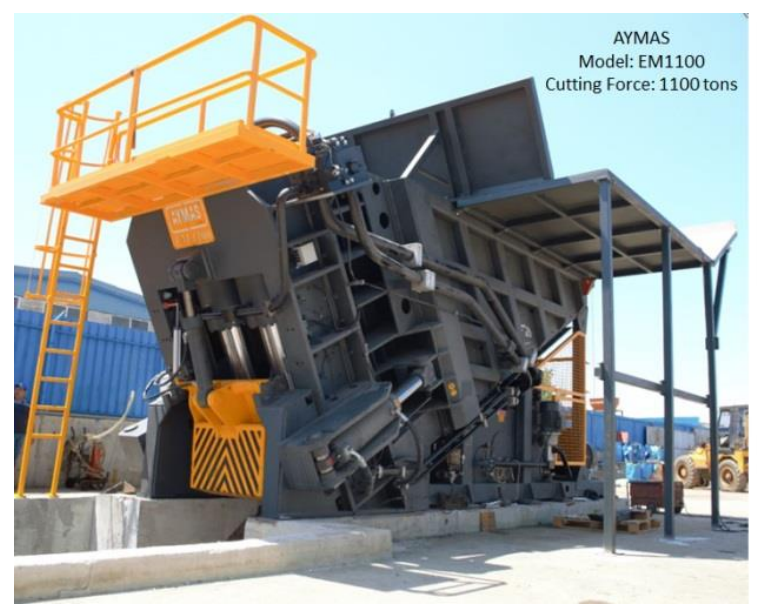

Fig. 1 Inclined scrap shear

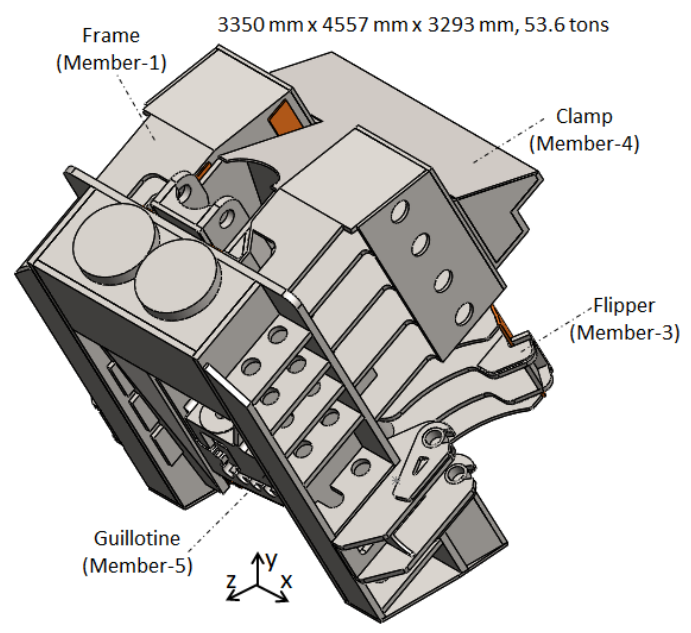

Fig. 2 SW analysis model

The analysis model consists of the members, which are frame (Member-1), two flippers (Member-2 and Member-3), clamp (Member-4), and guillotine (Member-5). There are a revolute joint between the flippers and the frame, a slider joint between the clamp and the frame, and a slider joint between the guillotine and the frame.

\section{FREE BODY DIAGRAMS OF MEMBERS AND SINGLE-BODY FE ANALYSIS}

The free-body diagrams of the moving members are shown in Fig. 3.

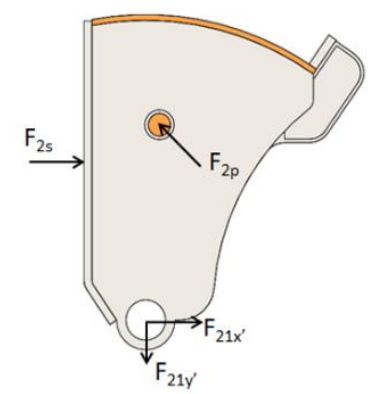

(a)

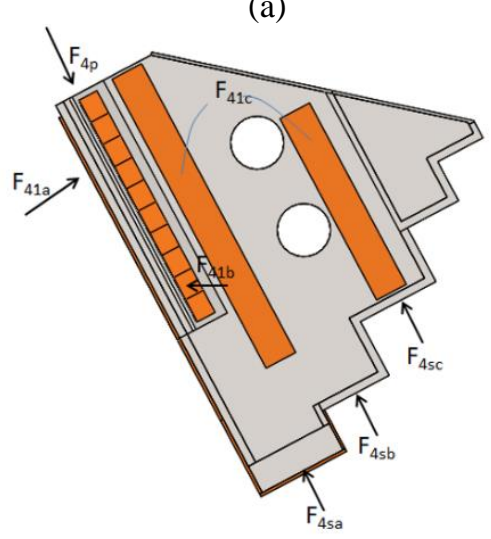

(b)

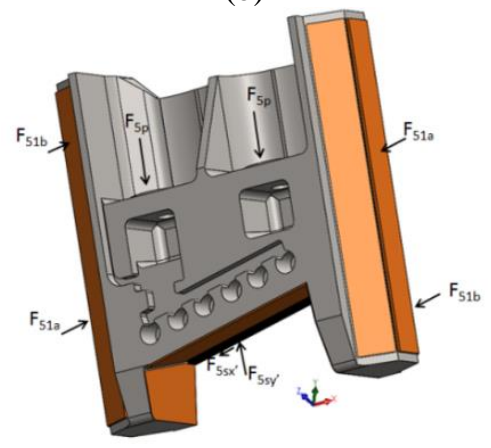

(c)

Fig. 3 Free-body diagrams of (a) flipper, (b) clamp, and (c) guillotine

$F_{2 p}$ in Fig. 2(a) is the pressure force of the hydraulic cylinder pushing the flipper. Knowing the maximum pressure as 300 bar and the diameter of the hydraulic piston, $F_{2 p}$ is calculated. The other forces $F_{21 x}, F_{21}$ ', and $F_{2 s}$ are calculated using the static equilibrium equations. $\mathrm{F}_{2 \mathrm{~s}}$ is the reaction force of the scrap, $F_{21 x}$, and $F_{21 y}$ are the components of the joint force between the flipper (Member-2) and the frame (Member-1).

$\mathrm{F}_{4 \mathrm{p}}$ in Fig. 2(a) is the pressure force of the hydraulic cylinder pushing the clamp. Knowing the maximum pressure as 300 bar and the diameter of the hydraulic piston, $F_{4 p}$ is calculated using the static equilibrium condition $\mathrm{F}_{4 \mathrm{sa}}+\mathrm{F}_{4 \mathrm{sb}}+\mathrm{F}_{4 \mathrm{sc}}=\mathrm{F}_{4 \mathrm{p}} . \mathrm{F}_{4 \mathrm{sa}}, \mathrm{F}_{4 \mathrm{sb}}$, and $\mathrm{F}_{4 \mathrm{sc}}$ are the reaction forces of the scrap, in the regions $a, b$, and $c$ respectively. The density of the scrap decrease from a to $b$, and to $c$; and the reaction forces decrease accordingly. An assumption is necessary to calculate the reaction forces. An initial guess can be as $F_{4 s a}=(16 / 21) F_{4 p}, F_{4 s b}=(4 / 21) F_{4 p}, F_{4 s c}=(1 / 21) F_{4 p} . F_{41 a}$ and $\mathrm{F}_{41 \mathrm{~b}}$ are calculated using the equilibrium equations. It is 
assumed that the reaction force of the scrap is symmetric and $\mathrm{F}_{41 \mathrm{c}}=0$.

Similarly, $F_{5 p}$ in Fig. 2(a) is the pressure force of the hydraulic cylinder pushing the guillotine. Knowing the maximum pressure as 300 bar and the diameter of the hydraulic piston, $\mathrm{F}_{5 \mathrm{p}}$ is calculated. The other forces in Fig. 1(c) are calculated using the static equilibrium equations considering $\mathrm{F}_{5 \mathrm{sx}},=\tan \left(12^{\circ}\right) \mathrm{F}_{5 \text { sy }}$, where $12^{\circ}$ is the slope of the upper blade.

The opposite of the forces shown in Fig. 3 act on the frame according to the law of action-reaction.

It should be noted that all the forces are distributed and the static equivalent of the forces are shown in the free-body diagrams. Assumptions are needed to define the distributions, which may be far from the realistic distribution, especially for the slider joints with large sliding surfaces $\left(F_{41}, F_{41 b}, F_{51 a}\right.$, $\mathrm{F}_{51 \mathrm{~b}}$ ).

It is possible to analyze the strength of the members separately by using single-body FE analysis using SW simulation. The static analysis is chosen. FE mesh is created for the members under study. The forces are defined. The inertia free solution is selected without any fixtures (boundary conditions). This method is not used in this study because it is more difficult compared with the multi-body FE analysis as used below.

\section{MULTI-BODY FE ANALYSIS}

It is possible to analyze the system in SW simulation by using the multi-body FE analysis. Here, the assembly of all the members is used. FE mesh of the assembly is created. The forces exerted on the scrap $\left(\mathrm{F}_{2 \mathrm{~s}}, \mathrm{~F}_{4 \mathrm{sa}}, \mathrm{F}_{4 \mathrm{sb}}, \mathrm{F}_{4 \mathrm{sc}}, \mathrm{F}_{5 \mathrm{sx}}, \mathrm{F}_{5 \mathrm{sy}}\right)$ are calculated by using the equilibrium equations, and it is assumed that they are uniformly distributed on the contacting faces. Only these forces are defined in the multi-body FE analysis. The other distributed joint forces in Fig. 3 are not defined and they are considered in SW by defining pin, rigid links, and no penetration between the contacting faces in the joints. The bottom face of the system is fixed to the ground. There are pin connections with rotation free options between the frame and flippers.

There are five hydraulic cylinders (HC) in the system: 2 HC-flipper, $1 \mathrm{HC}$-clamp, and $2 \mathrm{HC}$-guillotine. The HC's are evaluated by using conventional engineering methods and they are not considered in the FE analysis, and rigid links are defined to consider reaction forces at the connections of the HC's. The tubes and the piston rods of HC-flippers are joined to the frame and flippers by revolute joints, respectively. The tube of HC-clamp is joined to the frame by a revolute joint, and the piston rod of HC-clamp is joined to the clamp by a spherical joint. The tube of HC-guillotine is joined to the frame rigidly, and the piston rod of the HC-guillotine is joined to the guillotine rigidly. Rigid links are defined between $\mathrm{HC}$ tube and $\mathrm{HC}$ piston rod joints.

No penetration contacts are available for static, drop test, and nonlinear studies in SW (Solidworks, 2019). This contact type prevents interference between contacting faces but allows gaps to form. This is the most time-consuming option to solve. They are used for sliding surfaces in this study.

The information about the forces is given in Table 1 . The instant at which the cutting starts is considered for the positions of the members of the system. Gravity is not considered in this study.

TABLE 1 Forces for multi-body analysis

\begin{tabular}{|l|l|}
\hline Forces & Definitions and Values \\
\hline $\mathrm{F}_{2 \mathrm{~s}}$ & Calculated and defined as 1252.853 kN \\
$\mathrm{F}_{4 \mathrm{sa}}, \mathrm{F}_{4 \mathrm{sb}}, \mathrm{F}_{4 \mathrm{sc}}$ & Calculated and defined as 1723.388, 430.846, 107.711 kN \\
$\mathrm{F}_{5 \mathrm{sx}}, \mathrm{F}_{5 \mathrm{sy}}$ & Calculated and defined as 1014.169, 4771.293 kN \\
$\mathrm{F}_{2 \mathrm{p}}$ & Considered in rigid link definition for HC-flipper \\
$\mathrm{F}_{21 \mathrm{x}}, \mathrm{F}_{21 \mathrm{y}}$, & Considered in rotation free pin definition \\
$\mathrm{F}_{4 \mathrm{p}}$ & Considered in rigid link definition for the HC-clamp \\
$\mathrm{F}_{41 \mathrm{a}}$ & Considered in no penetration definition \\
$\mathrm{F}_{5 \mathrm{p}}$ & Considered in rigid link definition for the HC-guillotine \\
$\mathrm{F}_{51 \mathrm{a}}, \mathrm{F}_{51 \mathrm{~b}}$ & Considered in no penetration definition \\
\hline
\end{tabular}

Curvature-based mesh is used in SW. SW offers a default mesh size $(671 \mathrm{~mm})$. Approximately the half of the default value $(350 \mathrm{~mm})$ is selected at the initial analysis. The critical regions where high VonMises stresses are evaluated. Mesh control and smaller mesh sizes for critical regions or interpolation for singular results are applied if necessary.

\section{RESULTS AND DISCUSSIONS}

The initial results are shown in Fig. 4. The critical regions where high vonMises stresses result and their values are given in Table 2.

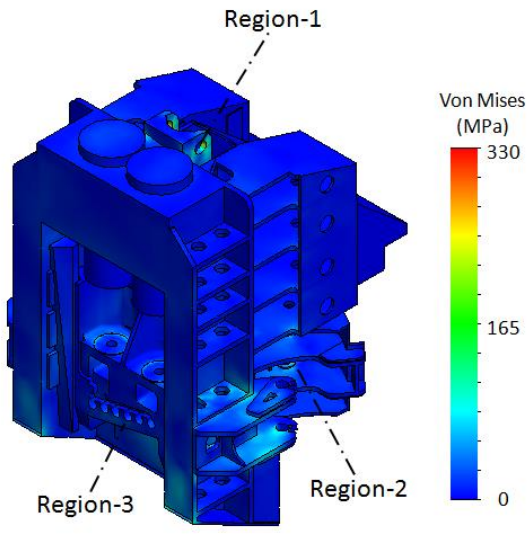

Fig. 4 Initial results for multi-body FE analysis

TABLE 2 Stresses at critical regions

\begin{tabular}{|c|c|c|}
\hline Region & Definition & $\begin{array}{c}\text { Value } \\
(\mathrm{MPa})\end{array}$ \\
\hline Region-1 & Bracket of revolute joint on frame for HC-clamp & 330 \\
Region-2 & Bracket of revolute joint on flipper for HC-flipper & 240 \\
Region-3 & Upper blade of guillotine & 210 \\
\hline
\end{tabular}

It is observed that the stress is very high for Region-1. Mesh control is used for Region-1 to control whether the result depends on the mesh size.

In Solidworks, only tetrahedral elements can be used for solid geometries. This element type is ideally composed of triangles with equal edge lengths. The ratio of the longest edge to the shortest edge is called the aspect ratio (Solidworks, 2019). The aspect ratio is an indicator of mesh quality. The tensile values in that element can deviate much from the actual, as the aspect ratio increases. Therefore, it may wrong to evaluate the maximum stress values in the analysis without considering mesh quality. 
After the mesh is obtained, the aspect ratio should not be greater than 1. This examination should be carried out especially in areas where the maximum stress occurs. In some geometries, geometric details can be ignored to improve mesh element quality, or mesh size can be decreased locally with mesh control. In the obtained stress distribution graph, color transitions should be soft and sudden color changes should be paid attention. In regions with sudden color change, the average stress value in the region where the maximum stress occurs should be evaluated instead of the maximum stress value.

The results depending on the mesh size are given Table 3 .

TABLE 3 Maximum stresses in Region-1 depending on the mesh size

\begin{tabular}{|c|c|c|c|c|}
\hline $\begin{array}{c}\text { Mesh } \\
\text { size } \\
(\mathrm{mm})\end{array}$ & $\begin{array}{c}\text { Maximum stress } \\
\text { in bushing hole } \\
(\mathrm{MPa})\end{array}$ & $\begin{array}{c}\text { Maximum stress } \\
\text { in the back area } \\
(\mathrm{MPa})\end{array}$ & $\begin{array}{c}\text { Total } \\
\text { number } \\
\text { of nodes }\end{array}$ & $\begin{array}{c}\text { Calculation } \\
\text { Time } \\
(\mathrm{Min})\end{array}$ \\
\hline 350 & 270 & 201 & 96495 & 8.4 \\
\hline 150 & 281 & 523 & 98967 & 10.5 \\
\hline 100 & 275 & 360 & 98932 & 10.8 \\
\hline 50 & 333 & 483 & 105828 & 13.1 \\
\hline 25 & 452 & 675 & 144433 & 19.5 \\
\hline
\end{tabular}

When the results in Table 3 are examined, it is seen that the tensile values obtained can vary greatly with mesh size. Only reducing the mesh size does not guarantee more accurate results. Here, mesh size as well as mesh quality is an important factor.

It is observed from Table 3 that there are unstable results due to singularity for the mesh size of $25 \mathrm{~mm}$ in the bushing hole. All the results in the back area are also unstable due to the singularities. The back area given in Table 3 means that the welded area between the frame and bracket carrying the clamp cylinder. The singularity is observed as high stress at single FE and the stresses are much lower at the adjacent FE's.

The maximum stress in Region- 1 can be evaluated as 275 MPa. The bracket material is St-52 and it is not suitable to exceed $210 \mathrm{MPa}$. The maximum pressure of 300 bar must be decreased or the thicknesses in the bracket must be increased.

Acknowledgements- This research was conducted in cooperation with AYMAS Ltd. and BLM Mekatronik Ltd. The authors would like to thank AYMAS Research and Development Center Engineers, Izmir-Turkey, for providing the necessary data for the investigation.

\section{REFERENCES}

[1] Aymas Recycling Machinery Inc. IS-Series Inclined Shear. 2019. http://www.aymasmakina.com/kategori/1-14/inclined-shears/.

[2] Bohdal L., Kukielka L., 2014. Application of variational and FEM methods to the modelling and numerical analysis of guillotining process for geometrical and physical nonlinearity, Mechanika 20(2): 197-204.

[3] Liu Y.Y., Wang Q., Yang J. S., and Xu J. S. 2009. Finite Element Analysis of Hydraulic Shears' Tool Carrier Based on ANSYS [J]. Modular Machine Tool \& Automatic Manufacturing Technique, 6.

[4] Metso Inc. N-Series Inclined Shear, 2019. https://www.metso.com/products/shears/n-series-inclined/.

[5] Ramamurti V., Sasikiran S., Kumar, P.V., 1997. Dynamic analysis of a guillotine-shearing machine, Journal of Materials Processing Technology 71: 202- 214

[6] Solidworks, 2019. https://help.solidworks.com/2019/english/solidworks/cworks/id_h elp_contact_pair.htm.

[7] Wang Q., Liu Y., Yang J., Xu, J., 2010. Finite Element Analysis and Structure Improvement of JCL-3× 1800 Hydraulic Shear's Frame [J]. Machine Tool \& Hydraulics, 18.

[8] Wisselink, H., Hue'tink, J. 2004. 3D FEM simulation of stationary metal forming processes with applications to slitting and rolling, Journal of Materials Processing Technology 148: 328-341. 